\title{
Questions and Answers on Practical Thrombotic Issues in SARS-CoV-2 Infection: A Guidance Document from the Italian Working Group on Atherosclerosis, Thrombosis and Vascular Biology
}

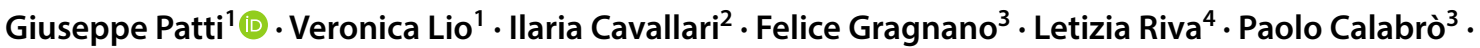 \\ Giuseppe Di Pasquale ${ }^{4}$. Vittorio Pengo ${ }^{5,6}$. Andrea Rubboli ${ }^{7}$ on behalf of the Italian Study Group on Atherosclerosis, \\ Thrombosis, Vascular Biology
}

Accepted: 1 October 2020 / Published online: 3 November 2020

(c) The Author(s) 2020

\begin{abstract}
In patients with coronavirus disease 2019 (COVID-19), the prevalence of pre-existing cardiovascular diseases is elevated. Moreover, various features, also including pro-thrombotic status, further predispose these patients to increased risk of ischemic cardiovascular events. Thus, the identification of optimal antithrombotic strategies in terms of the risk-benefit ratio and outcome improvement in this setting is crucial. However, debated issues on antithrombotic therapies in patients with COVID-19 are multiple and relevant. In this article, we provide ten questions and answers on risk stratification and antiplatelet/anticoagulant treatments in patients at risk of/with severe acute respiratory syndrome coronavirus 2 (SARS$\mathrm{CoV}-2$ ) infection based on the scientific evidence gathered during the pandemic.
\end{abstract}

Since December 2019, when the first cases of interstitial pneumonia were identified in the province of Wuhan, China, the infection due to severe acute respiratory syndrome coronavirus 2 (SARS-CoV-2) has rapidly involved a large part of the population worldwide, reaching the global pandemic level. National health systems had to implement public health responses to protect their populations and limit viral diffusion. In patients with coronavirus disease 2019 (COVID-19), the high prevalence of cardiovascular comorbidities [1] and the increased incidence of thrombotic events, sustained by multiple factors (Fig. 1), made complex and,

Giuseppe Patti

giuseppe.patti@uniupo.it

1 Department of Translational Medicine, "Maggiore della

Carità" Hospital, University of Eastern Piedmont, Via

Solaroli, 17, 28100 Novara, Italy

2 Campus Bio-Medico University of Rome, Rome, Italy

3 "Sant'Anna and San Sebastiano" Hospital of Caserta, University of Campania "Luigi Vanvitelli", Naples, Italy

4 "Maggiore" Hospital, Bologna, Italy

5 Campus Biomedico "Pietro d'Abano", University of Padua, Padua, Italy

6 Fondazione Arianna" On Anticoagulation, Bologna, Italy

7 “S. Maria delle Croci” Hospital, Ravenna, Italy

\section{Key Points}

Patients with coronavirus disease-19 (COVID-19) have an elevated risk of thrombotic events due to a high prevalence of cardiovascular risk factors, previous cardiovascular diseases and a pro-thrombotic status.

In patients with COVID-19 the thrombotic risk outweighs the bleeding risk.

Diagnostic and therapeutic approaches for thrombotic events in patients with COVID-19 should take into account the risk of contagion, the baseline risk profile and possible drug interactions.

at the same time, crucial the optimization of antithrombotic treatments. Moreover, guideline recommendations in this setting should be followed, taking into account peculiar features related to the pandemic, in particular, the modalities of transmission and the high contagiousness of SARS-CoV-2, with the related need for use of appropriate personal protective equipment and the design of specific in-hospital diagnostic and therapeutic protocols. 
Fig. 1 Pathogenesis of prothrombotic status and thrombotic complications in patients with COVID-19. COPD chronic obstructive pulmonary disease, COVID-19 coronavirus disease 2019, DIC disseminated intravascular coagulation

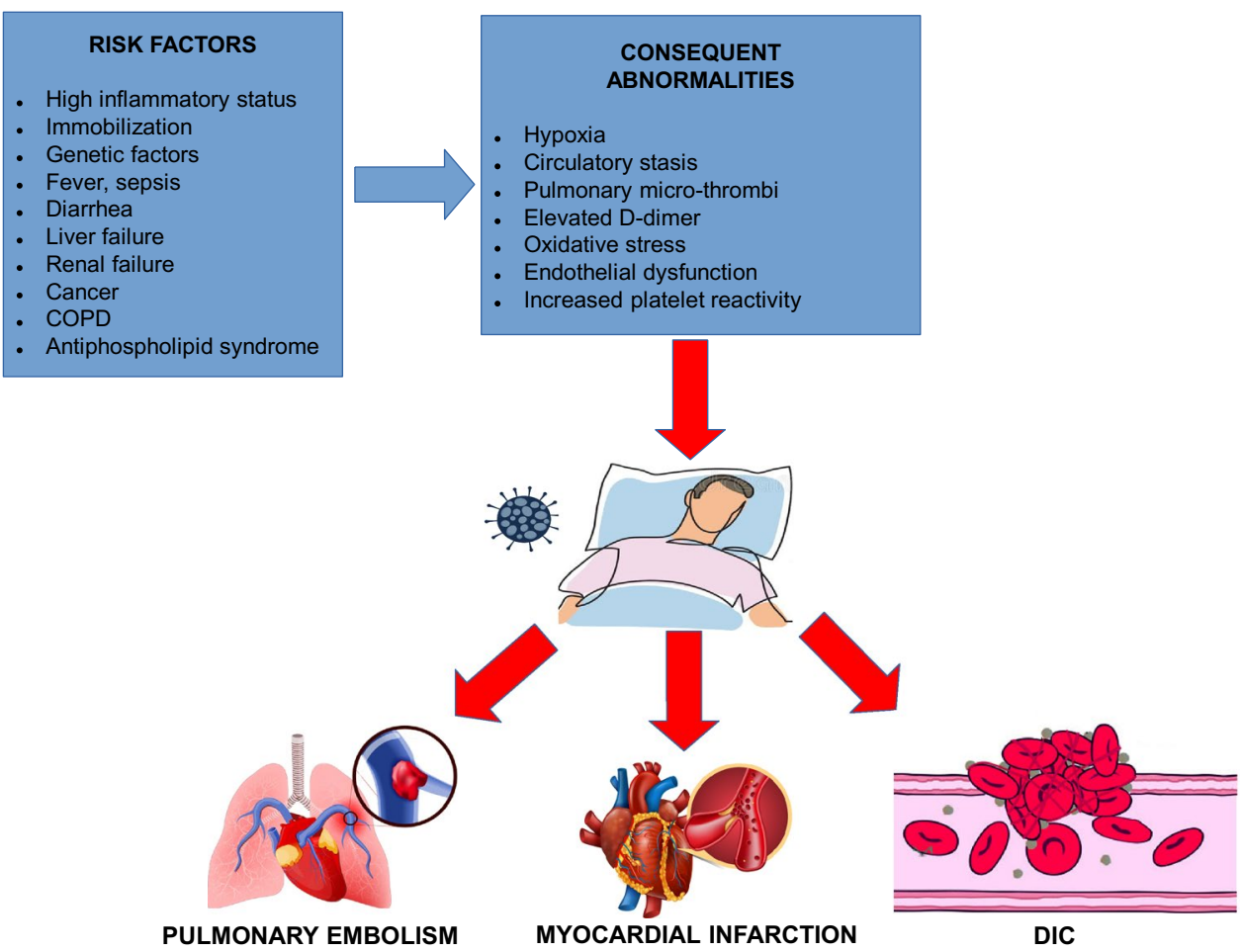

In this guidance document, we provide ten questions and answers on issues related to risk stratification and antithrombotic treatments in patients at risk of/with SARS-CoV-2 infection based on the scientific evidence gathered during the pandemic.

\section{What Advice Should We Give to Patients on Chronic Anticoagulant Therapy at Risk of SARS-CoV-2 Infection or with Mild COVID-19 Maintained at Home?}

In patients on oral anticoagulant therapy (OAC) who are at risk of contagion or have mild COVID-19 and are treated at home, anticoagulation should be individualized according to the patient's characteristics, clinical indication for OAC [atrial fibrillation (AF), venous thromboembolism (VTE), prosthetic heart valve], severity of infection, and therapies used for the viral infection. The duration of OAC for preventing AF-related thromboembolism is generally lifelong, based on the patient's $\mathrm{CHA}_{2} \mathrm{DS}_{2}$-VASc score ( $>1$ for males and $>2$ for females) [2]. Patients with VTE usually receive OAC for 3-6 months after the event, but treatment is frequently prolonged in the presence of relapsing episodes or a high risk of recurrence (i.e., idiopathic thromboembolism, cancer, antiphospholipid syndrome, and severe congenital thrombophilias) [3]. Patients with mechanical valve prostheses are given $\mathrm{OAC}$ for their lifetime, whereas OAC duration is limited to 3 months post-implantation in the case of a biological prosthesis.

During the pandemic, lockdown measures reducing interindividual contacts were applied in several countries to contain viral diffusion; thus, the indication was to stay home to keep safe, which was especially addressed to patients with more advanced age or comorbidities. This should be specifically applied to patients with AF or VTE, who are often older and have a higher prevalence of previous cardiovascular diseases or cancer. Moreover, health systems worldwide dealt with an emergency never seen before and had to limit elective, non-urgent, ambulatory visits. Therefore, in this context, the management of patients receiving vitamin $\mathrm{K}$ antagonist anticoagulants (VKAs) for AF or VTE was even more problematic. For these patients, it became difficult to access the centers for international normalized ratio (INR) control, especially if more frequent monitoring was needed because of unstable INR values (which is not uncommon). Moreover, during the outbreak, public centers for INR control had a reduced number of staff members due to the redistribution of care workers for the emergency and re-addressed or limited the outpatient flow to contain the viral diffusion as much as possible. Finally, general practitioners were not able to immediately assist patients on VKAs in need of INR adjustments, as they were also fully involved in the management of COVID-19 patients.

Accordingly, in patients at risk for SARS-CoV-2 infection on VKAs and who have substantial "stability" in their INR 
Table 1 Indications for patients at risk of/with SARS-CoV-2 infection maintained at home

\section{Patients on VKAs at risk of SARS-CoV-2 infection}

If INR values are stable (i.e., time in therapeutic range $>60 \%$ ), a prolongation of the INR control intervals may be considered (every 4-8 weeks)

The use of portable coagulometer devices with self-measurement of INR is encouraged

Switching from VKAs to DOACs must be considered

In the case of unstable INR values, switching from VKAs to DOACs is recommended

\section{Patients on VKAs with mild COVID-19 maintained at home}

The use of portable coagulometer devices with self-measurement of INR is encouraged

Switching from VKAs to DOACs must be considered, taking into account possible drug interactions

In the case of unstable INR values, switching from VKAs to DOACs is recommended

Patients not on oral anticoagulant therapy with asymptomatic SARS-CoV-2 infection

No thromboprophylaxis is indicated

Patients not on oral anticoagulant therapy with mild COVID-19

Thromboprophylaxis with LMWH is indicated if multiple risk factors for VTE are present and bleeding risk is low

COVID-19 coronavirus disease 2019, DOAC direct oral anticoagulant, INR international normalized ratio, LMWH low-molecular weight heparin, SARS-CoV-2 severe acute respiratory syndrome coronavirus 2, VKA vitamin $\mathrm{K}$ antagonist anticoagulant, VTE venous thromboembolism

\begin{tabular}{|c|c|c|c|c|c|c|c|c|c|c|c|c|c|c|c|c|}
\hline & & \multicolumn{4}{|c|}{ DOACs } & \multicolumn{2}{|c|}{ VKAs } & \multicolumn{4}{|c|}{ Heparins } & \multicolumn{5}{|c|}{ ANTIPLATELET } \\
\hline & & 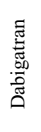 & 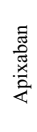 & 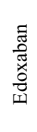 & 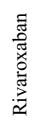 & 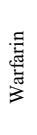 & 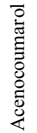 & 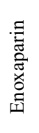 & 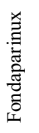 & 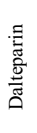 & $\overrightarrow{⿷ 匚 ⿳}$ & 言 & 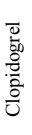 & 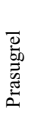 & 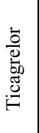 & 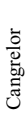 \\
\hline \multirow{10}{*}{ 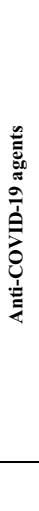 } & (Hydroxy)chloroquine & $\uparrow$ & & $\uparrow$ & & & & & & & & & & & & \\
\hline & Azithromycin & $\uparrow$ & $\uparrow$ & $\uparrow \uparrow$ & $\uparrow$ & $\uparrow \uparrow \uparrow$ & & & & & $\uparrow \uparrow \uparrow$ & & & & & \\
\hline & Lopinavir/ritonavir & $\uparrow \uparrow \uparrow$ & $\uparrow \uparrow \uparrow$ & $\uparrow \uparrow \uparrow$ & $\uparrow \uparrow \uparrow$ & $\downarrow \downarrow$ & $\downarrow \downarrow$ & & & & & & $\downarrow \downarrow$ & $\downarrow$ & $\uparrow \uparrow$ & \\
\hline & Atazanavir & $\uparrow \uparrow \uparrow$ & $\uparrow \uparrow \uparrow$ & $\uparrow \uparrow \uparrow$ & $\uparrow \uparrow \uparrow$ & $\uparrow$ & & & & & & & $\downarrow \downarrow$ & $\downarrow$ & $\uparrow \uparrow$ & \\
\hline & Ribavirin & & & & & $\downarrow \downarrow$ & & & & & & & & & & \\
\hline & Remdesivir & & & & & & & & & & & & & & & \\
\hline & Tocilizumab & & $\downarrow$ & & $\downarrow$ & $\downarrow$ & $\downarrow$ & & & & & & $\downarrow$ & $\downarrow$ & $\downarrow$ & \\
\hline & Interferon- $\beta$ & & & & & & & & & & & & & & & \\
\hline & Methylprednisolone & & & & & $\downarrow \downarrow$ & & & & & $\downarrow$ & & & & & \\
\hline & Paracetamol & & & & & $\uparrow \uparrow$ & $\uparrow \uparrow$ & & & & & & & & & \\
\hline
\end{tabular}

Fig. 2 Interactions between antithrombotic drugs and agents used for COVID-19. White: no data; green: no interaction; yellow: minor interaction, possibly requiring dose reduction of DOACs, additional INR controls (if VKA therapy) or functional monitoring of antiplatelet activity (in the case of $\mathrm{P} 2 \mathrm{Y}_{12}$ treatment); red: co-administration is contraindicated because of significant interaction. Upward arrows: increased activity of the antithrombotic drug, proportional to the number of arrows; downward arrows: decreased activity of the antithrombotic drug, proportional to the number of arrows. Adapted from ESC Guidance for the Diagnosis and Management of CV Disease during the COVID-19 Pandemic (https://www.escardio.org/ Education/COVID-19-and-Cardiology/ESC-COVID-19-Guidance). COVID-19 coronavirus disease 2019, CV cardiovascular, DOAC direct oral anticoagulant, ESC European Society of Cardiology, INR international normalized ratio, $U F H$ unfractionated heparin, $V K A$ vitamin $\mathrm{K}$ antagonist anticoagulant values within the therapeutic range (i.e., time in therapeutic range $>60 \%$ ), a prolongation of the INR control intervals may be considered (every 4-8 weeks) [4] (Table 1). The self-measurement of INR values by portable coagulometer devices is encouraged [5]. Switching from VKAs to direct oral anticoagulants (DOACs) must be considered [6]. 
In patients with mild COVID-19 treated at home who are on VKAs, the use of portable coagulometer devices should be implemented [7]. Moreover, switching from VKAs to DOACs has a clear indication. In fact, the duration of home isolation can be longer than 4 weeks; INR values are generally unstable during acute infection or illness; and paracetamol decreases the synthesis of vitamin K-dependent clotting factors [8], and therefore there is a major interaction between VKAs and this drug largely utilized for COVID-19-related fever (Fig. 2).

\section{What are the Relevant Interactions Between Anticoagulant Drugs and Anti-SARS-CoV-2 Agents?}

Treatment approaches in patients with COVID-19 include a combination of several drugs with virtually synergistic effects acting at different steps of the pathogenetic mechanisms of the disease [9]. Scientific evidence in this regard is currently limited but in continuous and rapid evolution [10]. In the first period of the SARS-CoV-2 pandemic, a widespread practice pattern was to treat with hydroxychloroquine both patients with mild COVID-19 kept at home and those with more severe disease requiring hospitalization. Notably, most recent data shifted the therapeutic paradigm from the initial enthusiasm to a rise of skepticism and uncertainty when more in-depth analyses of the use of hydroxychloroquine emerged [9-11]. In particular, the World Health Organization prematurely stopped the hydroxychloroquine arm of the Solidarity trial (NCT04330690), where no survival benefit had been observed in patients hospitalized for COVID-19. However, no significant interaction has been described between hydroxychloroquine and any antithrombotic agent (Fig. 2). The protease inhibitors lopinavir and atazanavir (either drug given in association with ritonavir or cobicistat) [12-15] have been largely utilized; however, accumulating data from recently published randomized studies and an interim analysis of the previously mentioned Solidarity trial, showed no reduction in mortality with the use of lopinavir/ritonavir in COVID-19 [16]. Furthermore, the nucleotide analog remdesivir [17-19] (developed for the treatment of Ebola and Marburg virus infections) showed favorable results in hospitalized patients with SARS-CoV-2 infection [20]. A less robust clinical benefit has been demonstrated with the nucleotide analog ribavirin [21], as well as with azithromycin [22], a macrolide often administered for COVID-19 in combination with hydroxychloroquine. In particular, the effects of the drug combination azithromycin plus hydroxychloroquine are controversial, given the limited evidence, the significant biases of available studies, and the pending results of randomized studies [22]. Interferon- $\beta$, currently authorized for the treatment of multiple sclerosis, was used in the treatment of Middle East respiratory syndrome coronavirus (MERS-CoV) infections with some positive preclinical results [14]; on this basis, it has been now included in anti-SARS-CoV-2 experimental protocols. Finally, due to recent encouraging clinical results, the use of glucocorticoids, mainly dexamethasone [23], and/ or tocilizumab [24] (an interleukin-6 inhibitor) in patients with severe COVID-19 has increased. In asymptomatic or pauci-symptomatic patients with SARS-CoV-2 infection maintained at home, during the initial phases of the outbreak, treatment with hydroxychloroquine (with or without azithromycin) was generally performed, although the diffusion of this approach was greatly different across different countries.

There is no significant interaction between all agents used in COVID-19 and low-molecular weight heparins (LMWHs) or fondaparinux (Fig. 2) [25].

Ribavirin and lopinavir/ritonavir decrease the anticoagulant effects of VKAs, especially of warfarin, by interfering with cytochrome P450 3A4 (CYP3A4). Azithromycin significantly increases the anticoagulant action of warfarin; thus, co-administration should be avoided or at least INR monitoring must be intensified. Furthermore, azithromycin enhances the anticoagulant effects of unfractionated heparin (UFH) (Fig. 2).

DOACs, except dabigatran, are metabolized by CYP3A4. All DOACs are substrates of intestinal P-glycoprotein. In specific cases of uncertainty about possible drug interference, although not routinely recommended, a measurement of DOAC concentration could be considered. Macrolides inhibit the action of intestinal P-glycoprotein, resulting in a potential increase in the blood levels of DOACs and increased hemorrhagic risk. To date, no clinically relevant interference has been reported for dabigatran, rivaroxaban, or apixaban and azithromycin. There are no data on the interaction between azithromycin and edoxaban. Accordingly, the labeling of this agent does not recommend reducing the edoxaban dose in the case of coadministration. However, such interaction has to be considered plausible, similarly to what occurs for edoxaban with clarithromycin or erythromycin [25]. Indeed, in the randomized VTE-CANCER study [26], where edoxaban was compared to dalteparin in patients with cancer and VTE, the concomitant use of azithromycin required reduction of the DOAC dose (Fig. 2).

Some antiviral agents used for COVID-19 (lopinavir/ ritonavir, atazanavir) are competitors of intestinal P-glycoprotein and inhibit CYP3A4 [9]. These agents significantly increase the bleeding risk of DOACs, due to an elevation in blood concentrations [27]. Thus, an absolute contraindication for co-administering the aforementioned antiviral substances with DOACs must be indicated (Fig. 2). Although dabigatran is not metabolized by CYP3A4, this 
drug is a substrate of intestinal P-glycoprotein; therefore, the concomitant use of those antiviral agents and dabigatran should be reasonably prohibited. Accordingly, in COVID-19 patients on DOACs with an established indication for lopinavir/ritonavir or atazanavir, DOACs will be temporarily stopped and replaced with LMWH over the short term [25]. No significant interaction between DOACs and ribavirin is described.

No significant interference has been reported between VKAs or DOACs and interferon- $\beta$ or tocilizumab (Fig. 2). However, the use of this monoclonal antibody has been associated with hepatotoxicity [28]. Thus, close monitoring of liver function and coagulation indices is mandatory if tocilizumab is given in patients on anticoagulant treatment. Furthermore, as tocilizumab may cause an increase in the expression of CYP3A4, its co-administration can lead to a slight decrease in blood levels of anti-Xa agents (especially apixaban and rivaroxaban); therefore, close functional monitoring of coagulation parameters is indicated [25].

\section{What to Do in Patients on Antithrombotic Agents Hospitalized for COVID-19?}

Therapy with non-steroidal anti-inflammatory drugs (NSAIDs) was hypothesized to be a potential risk factor for a more serious clinical presentation of COVID-19, but these data have not been confirmed in the most recent series [29]. Aspirin at the "antiplatelet" daily dose of 75-100 mg has limited anti-inflammatory effects. Given its significant cardiovascular benefits, in patients on chronic therapy with aspirin for secondary prevention who are hospitalized or are maintained at home for COVID-19, this agent must be continued (Table 2), consistent with the recent recommendations by McCullough and colleagues
[30]. Aspirin treatment for primary cardiovascular prevention should also be continued, unless contraindications have occurred during in-hospital stay (acute liver failure, severe renal failure, severe thrombocytopenia, documented drug interactions, planned invasive procedures). In patients hospitalized for COVID-19, dual antiplatelet therapy (aspirin plus oral $\mathrm{P} 2 \mathrm{Y}_{12}$ receptor inhibitor) must be continued in those who have recently undergone percutaneous coronary intervention (PCI) (within $\leq 3$ months), unless hemorrhagic events are reported [25].

Regarding $\mathrm{P}_{2} \mathrm{Y}_{12}$ receptor inhibitors, lopinavir/ritonavir and atazanavir reduce the antiplatelet effect of clopidogrel and prasugrel (the latter not significantly), while they increase blood concentrations of ticagrelor (Fig. 2) [9]. Thus, in this setting, the co-administration of aspirin plus prasugrel should be preferred. Indeed, the most recent evidence has indicated no clinical benefit of lopinavir/ ritonavir, and the use of such antiviral drugs has progressively declined over the pandemic course [31]. However, in patients with COVID-19 who are candidates for lopinavir/ritonavir or atazanavir treatment and are on aspirin plus clopidogrel for a recent ( $\leq 3$ months) PCI after chronic coronary syndrome, clopidogrel is continued, with blood cell count and ischemic event monitoring (Table 2). In patients with COVID-19 who are candidates for lopinavir/ ritonavir or atazanavir and are on aspirin plus clopidogrel/ ticagrelor for a recent ( $\leq 3$ months) PCI after acute coronary syndrome (ACS), the replacement of clopidogrel/ ticagrelor with prasugrel is indicated. If prasugrel is contraindicated, therapy with clopidogrel/ticagrelor is continued, with blood cell count and ischemic/bleeding event monitoring. There is no significant interference between clopidogrel/ticagrelor/prasugrel and the other agents used in COVID-19.

Table 2 Indications for patients receiving chronic antithrombotic treatments admitted for COVID-19

\section{Patients on chronic antiplatelet therapy}

Aspirin therapy for primary cardiovascular prevention should be continued, unless contraindications have arisen or there is need for venous thromboprophylaxis

Antiplatelet therapy for secondary cardiovascular prevention must be continued, considering possible drug interactions

Dual antiplatelet therapy in patients who have undergone PCI within $\leq 3$ months must be continued unless hemorrhagic events are reported

In patients on aspirin plus clopidogrel/ticagrelor who have undergone a recent PCI ( $\leq 3$ months) for ACS requiring treatment with lopinavir/ ritonavir or atazanavir, switching from clopidogrel/ticagrelor to prasugrel is indicated. If prasugrel is contraindicated, therapy with clopidogrel/ticagrelor is continued, monitoring blood cell count and ischemic/bleeding events

In patients on aspirin plus clopidogrel who have undergone a recent PCI ( $\leq 3$ months) for stable coronary syndrome requiring treatment with lopinavir/ritonavir or atazanavir, clopidogrel is continued, monitoring blood cell count and ischemic events

No significant interaction between clopidogrel/prasugrel/ticagrelor and the other agents used for COVID-19 are present

\section{Patients on chronic OAC}

If indication for OAC is adequate and no contraindication exists, short-term switching from OAC to LMWH is reasonable

$A C S$ acute coronary syndrome, COVID-19 coronavirus disease 2019, LMWH low-molecular weight heparin, $O A C$ oral anticoagulant therapy, $P C I$ percutaneous coronary intervention 
Finally, in patients on chronic OAC, if the indication for $\mathrm{OAC}$ is adequate and no contraindication exists, short-term switching from OAC to LMWH is reasonable (Table 2).

\section{Antithrombotic Prophylaxis in Patients with SARS-CoV-2 Infection: When and How?}

Thromboprophylaxis is not recommended in asymptomatic patients in whom a nasopharyngeal swab tested positive for molecular detection of SARS-CoV-2 RNA. In patients with mild COVID-19 maintained at home, thromboprophylaxis is indicated only if multiple risk factors for VTE and a low bleeding risk exist (Table 1) [25, 30].

In patients with more severe COVID-19, the release of pro-inflammatory mediators, platelet activation, oxidative stress, endothelial dysfunction [32], prolonged immobilization, hypoxia, circulatory stasis, the use of mechanical ventilation, liver dysfunction, central venous catheters, and nutritional deficit increase the risk of VTE (Fig. 1). Thus, these patients should be stratified upon hospitalization based on the clinical pattern and on the risk of thrombotic and bleeding complications. However, it is worth pointing out that this stratification is a dynamic process requiring a periodical reassessment according to clinical course and laboratory tests. Patients admitted to the intensive care unit (ICU), regardless of the need for mechanical ventilation, and those with acute respiratory insufficiency must be considered at high thromboembolic risk; thus, they require thromboprophylaxis $[33,34]$. In patients with mild-tomoderate respiratory symptoms, with or without evidence of interstitial pneumonia, stratification based on the risk of VTE assessed using the PADUA Prediction Score [35] or the IMPROVE VTE score [36] is recommended (Fig. 3). A PADUA score of $\geq 4$ identifies patients with an elevated risk of VTE; an IMPROVE score of 2-3 indicates an intermediate risk, whereas a value of $\geq 4$ indicates a high risk. Following this stratification, thromboprophylaxis is not indicated in patients with a PADUA score of $<4$ or an IMPROVE score of 1 . In patients with a PADUA score indicating a high risk or an IMPROVE score indicating intermediate-to-high risk, thromboprophylaxis is needed, if contraindications do not exist.

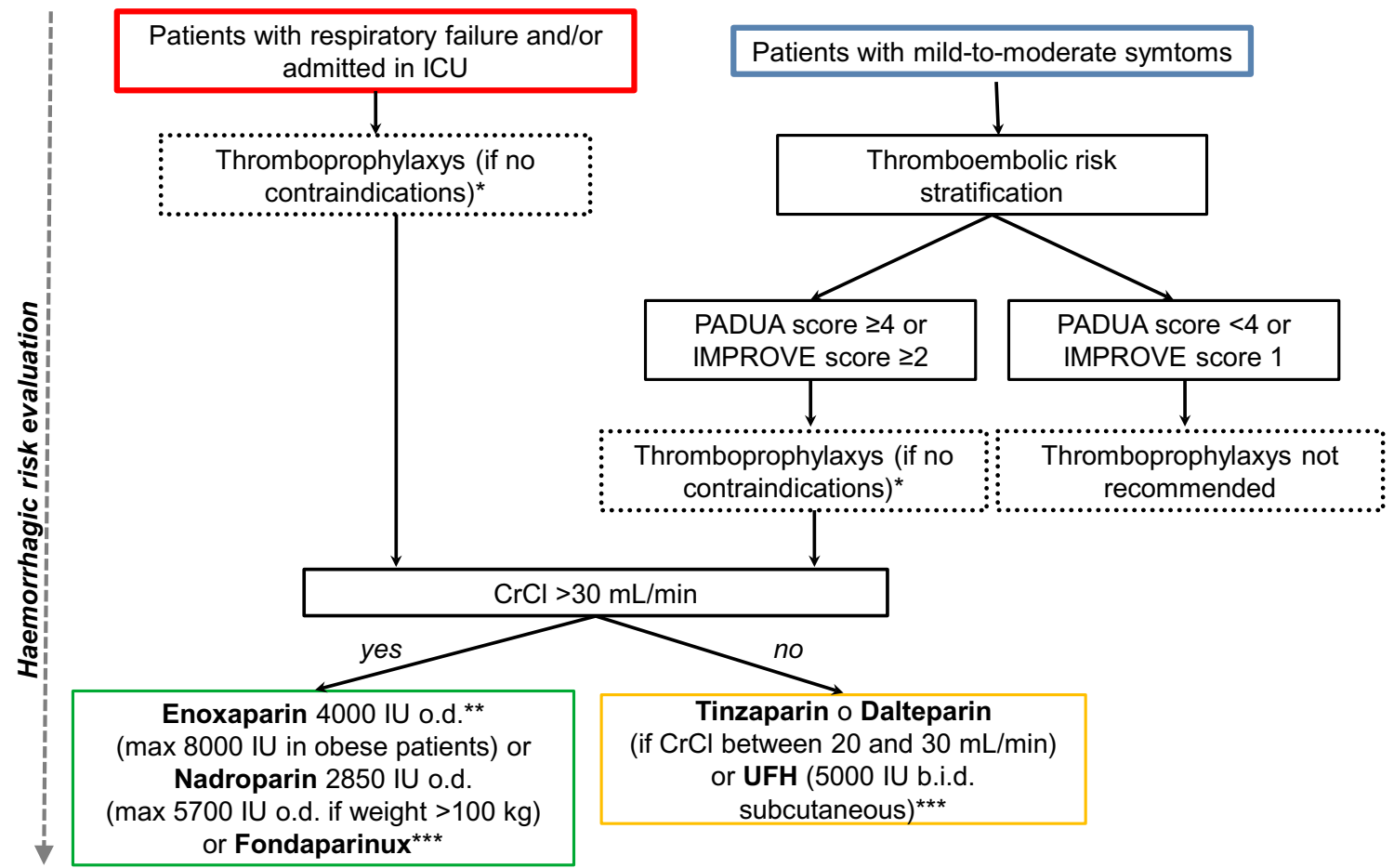

* If pharmacological prophylaxys is contraindicated, it is possible to perform mechanical thromboprophylaxys by intermittent mechanical elasto-compression

** Due to recent reports showing in COVID-19 a pro-thrombotic milieu and high rates of venous thromboembolism, the use of higherthan-prophylactic doses of enoxaparin (e.g. 4000 IU BID) has been recently encouraged

*** In patients at high thrombotic risk (PADUA score $\geq 4$ or IMPROVE score $\geq 4$ ) and low bleeding risk, consider thromboprophylaxys up to 45 days after discharge

Fig. 3 Flowchart for thromboprophylaxis in patients with COVID-19. COVID-19 coronavirus disease 2019, CrCl creatinine clearance, BID/b.i.d. twice a day $I C U$ intensive care unit, $I U$ international units, o.d. every day, $U F H$ unfractionated heparin 
The choice of agent for thromboprophylaxis should take into account renal function. In patients with creatinine clearance $<30 \mathrm{~mL} / \mathrm{min}$, UFH [5000 international units (IU) twice a day (BID) subcutaneously] can be used, whereas in those with creatinine clearance $20-30 \mathrm{~mL} / \mathrm{min}$, tinzaparin or dalteparin represents an option [25]. Notably, measurement of plasma anti-Xa activity is recommended in patients with renal failure, adjusting the dosage to maintain levels of $0.2-0.5 \mathrm{U}$ anti-Xa/mL. In patients with creatinine clearance $>30 \mathrm{~mL} / \mathrm{min}$, enoxaparin is the first choice for thromboprophylaxis, with nadroparin or fondaparinux alternatively. Regarding the dosage of thromboprophylaxis drugs, there is an extensive debate. The World Health Organization expressed an orientation in favor of using a daily prophylactic dose of LMWH. A general indication is to give enoxaparin at a daily dose of $4000 \mathrm{IU}$ (increased up to $8000 \mathrm{IU}$ in obese patients) or nadroparin [37].

Notably, available data on the clinical benefit of thromboprophylaxis in patients with COVID-19 were initially based mainly on a single analysis of approximately 100 patients included in a retrospective study. Here, prophylaxis was associated with lower mortality only in patients having criteria for sepsis-induced coagulopathy or with D-dimer values sixfold higher than the reference limit [38]. Furthermore, data from a large United States cohort suggested that systemic anticoagulation (including oral, subcutaneous, or intravenous forms) may be associated with higher survival among patients hospitalized with COVID-19 [39]. Notably, in agreement with recent reports showing in such patients a pro-thrombotic milieu [32] and high rates of venous thromboembolic complications, the use of intermediate doses of enoxaparin (e.g., 4000 IU BID) for thromboprophylaxis has been recently encouraged, as prophylactic doses were considered insufficient for this aim [25]. However, specific studies are urgently needed to establish the optimal approach to thromboprophylaxis with LMWH in this setting, but, in the absence of robust evidence supporting higher dosing regimens, if thromboprophylaxis is indicated, it appears reasonable to utilize LMWH at prophylactic dosages. There are no specific data on the extension of thromboprophylaxis beyond hospitalization in patients with COVID-19; it is advisable to proceed individually and consider this extension up to a maximum of 45 days after discharge only in patients with a high VTE risk and a low risk of bleeding [25].

\section{What is the Approach for Diagnosing Venous Thromboembolism in Patients with COVID-19?}

The diagnosis of pulmonary embolism (PE) in patients with SARS-CoV-2 infection must be based on the integration of clinical, laboratory, and instrumental data (Table 3). D-dimer testing has a central role as a diagnostic marker for VTE; monitoring can allow an early diagnosis, but at the same time, it may be non-specific, if not contextualized. Indeed, over $50 \%$ of patients admitted for COVID-19 present elevated D-dimer values (in most cases $<1.0 \mathrm{mcg} / \mathrm{mL}$ ), even without thrombotic complications [40]. Thus, in line with the diagnostic algorithms provided by pre-existing guidelines [41], D-dimer must be measured only in the presence of a clinical suspicion of VTE. Conversely, in the absence of concomitant clinical worsening, high levels of D-dimer should not guide diagnostic and therapeutic processes. PE is suspected if increased values of D-dimer are associated with clinical symptoms/signs of deep venous thrombosis (DVT), worsening hypoxemia disproportionate to the degree of respiratory involvement, and/or acute right ventricular dysfunction.

Table 3 Indications for the management of VTE in patients with COVID-19

In patients with a worsening clinical status, especially in those without anticoagulant treatment, a diagnosis of VTE must always be suspected In patients with suspected VTE, the diagnostic and therapeutic workup must integrate clinical data, laboratory findings, and imaging test results Measurement of D-dimer for diagnosing VTE must be performed only if a clinical suspect exists

Vascular/cardiac ultrasound imaging for diagnosing VTE should precede radiological imaging

Patients undergoing a $C T$ scan for worsening respiratory status should receive angio-CT sequences to exclude PE

The use of LMWH for treating a VTE episode is preferred. UFH should be limited to patients with $\mathrm{CrCl}<30 \mathrm{~mL} / \mathrm{min}$

An invasive "catheter"-based therapy for PE is indicated in selected cases with contraindication to anticoagulant drugs, recurrent events despite adequate anticoagulation, or when systemic fibrinolysis cannot be performed

For the risk stratification of patients with VTE, monitoring of the following parameters is useful: troponin, BNP, D-dimer, blood cell count, fibrinogen, prothrombin time, activated partial thromboplastin time, and degradation products of fibrin

After the initial approach, DOACs may represent an option for in-hospital treatment of a VTE episode in patients with clinical stability and decreasing inflammation

After a VTE episode, DOACs should represent the therapy of choice at discharge

$B N P$ brain natriuretic peptide, COVID-19 coronavirus disease 2019, $C r C l$ creatinine clearance, $C T$ computed tomography, DOAC direct oral anticoagulant, $L M W H$ low-molecular weight heparin, $P E$ pulmonary embolism, $U F H$ unfractionated heparin, VTE venous thromboembolism 
The use of imaging techniques in diagnosing a VTE episode is complex, because of the risk of viral transmission to other patients and to healthcare workers, and must be regulated by specific in-hospital protocols aimed at limiting such risk. Each imaging test must be subsequent to an integrated evaluation of clinical and laboratory data. Notably, vascular or cardiac ultrasound examination usually anticipates radiological imaging and requires appropriate personal protective equipment and specific methods for sanitizing the instruments. In view of the high rates of DVT in ICU patients, an extensive use of vascular ultrasound is advisable to assess broadly the diagnosis of DVT. Due to the higher risk of PE, patients with COVID-19 undergoing a lung computed tomography (CT) scan for worsening respiratory function should receive angio-CT sequences to optimize the diagnostic process [42]. In patients with acute respiratory distress syndrome (ARDS) and suspected PE, performing radiological tests is difficult because of patient's prone position and unstable clinical conditions. Here, echocardiographic evidence of deterioration of the right ventricular function or (more rarely) of transit thrombus represents a relevant finding justifying further diagnostic steps and on this basis the initiation of specific treatments.

\section{How to Stratify the Prognosis of Patients with COVID-19?}

Prognostic stratification of patients with COVID-19 is important and may guide treatment modalities. A higher inflammatory status (as identified by increased C-reactive protein levels or neutrophil/lymphocyte ratio) has been correlated with a poorer outcome, including lower survival [43]. The measurement of troponin and brain natriuretic peptide (BNP) is also useful, as an increase in these parameters indicates acute myocardial damage and hemodynamic overload $[33,41,44]$. Various abnormalities in the hemostatic parameters were associated with a higher risk of cardiovascular complications, mechanical ventilation, and mortality, in particular, thrombocytopenia (especially $<50,000 \times 10^{9} / \mathrm{L}$ ) and elevation of D-dimer ( $>3 \mu \mathrm{g} / \mathrm{mL})$, fibrinogen $(>1 \mathrm{~g} / \mathrm{L})$, or degradation products of fibrin. Recent studies suggested a relationship between severity in the clinical course of COVID-19 and spontaneous prothrombin time prolongation (>3 s) or activated partial thromboplastin time prolongation (>5 s) [33, 41, 45]. Accordingly, all the aforementioned hemostatic markers should be routinely monitored in these patients, with the aim to obtain a more accurate prognostic stratification.

\section{How to Manage Antithrombotic Treatment in Patients with COVID-19 Complicated by Venous Thromboembolism?}

In COVID-19 patients with VTE, the indications for treatment are based on pre-existing guidelines and should be integrated with specific assessments related to the SARSCoV-2 infection [40]. As described above, the choice of the therapeutic regimen must also take into account considerations regarding the severity of clinical presentation, co-existence of renal disease, liver dysfunction and/or thrombocytopenia, and possible drug interactions with antiviral drugs [33]. The use of parenteral anticoagulants represents the best initial option, due to the greater manageability and the lower risk of drug interference (Table 3). LMWH at anticoagulant dosages (i.e., subcutaneous enoxaparin $1 \mathrm{mg} / \mathrm{kg}$ BID or nadroparin 86 IU/kg BID) should be preferred over UFH, which exposes healthcare workers to an increased risk of contagion, due to frequent blood samplings for dose adjustment. UFH should be used only in patients with creatinine clearance $<30 \mathrm{~mL} / \mathrm{min}$. An invasive catheter-based therapy for PE (locoregional thrombolytic therapy or embolectomy) is indicated in selected cases with contraindication to anticoagulant drugs, in those who experience recurrent events despite adequate anticoagulation, or when systemic fibrinolysis cannot be performed.

After the initial approach with LMWH, DOACs may represent an option for in-hospital treatment of a VTE episode only in patients with clinical stability and decreasing inflammation (Table 3). Unless contraindicated, DOACs should be preferred over VKAs, given they are easier to manage during the in-hospital stay and transition to the subsequent home regimen. However, as mentioned above, the choice of the OAC drug must consider possible interactions with anti-SARS-CoV-2 drugs [33, 39].

\section{How to Manage Anticoagulant Therapy in Patients Hospitalized for COVID-19 Who Develop Atrial Fibrillation?}

In patients with COVID-19, the onset or recurrence of AF is favored by fever, hypoxia, and adrenergic activation due to respiratory failure $[46,47]$. Here, anticoagulant treatment for preventing thromboembolic events must be guided by the $\mathrm{CHA}_{2} \mathrm{DS}_{2}$-VASc score rather than the characteristics of the arrhythmic episodes (i.e., duration of the episodes, number of relapses). It is reasonable to initiate anticoagulation with LMWH and then switch to OAC during the hospitalization 
Table 4 Criteria of the ISTH for the diagnosis of disseminated intravascular coagulation

Evaluation of the risk of DIC

ISTH score

Coagulation parameters

Diagnostic score

ISTH score calculation
Does the patient present a clinical condition consistent with DIC?

No: do not proceed with the score

Yes: proceed with the score

Prothrombin time, platelet count, D-dimer, degradation products of fibrin, fibrinogen

Prolongation of prothrombin time: $\leq 3 \mathrm{~s}=0$ points; $>3-\leq 6 \mathrm{~s}=1$ point; $>6 \mathrm{~s}=2$ points Platelets: $\geq 100 \times 10^{9} / \mathrm{L}=0$ points $;<100 \times 10^{9} / \mathrm{L}=1$ point $;<50 \times 10^{9} / \mathrm{L}=2$ points

D-DIMER, DEGRADATION PRODUCTS OF FIBRIN: normal $=0$ points; moderately increased $=2$ points; markedly increased $=3$ points

Fibrinogen: $>1 \mathrm{~g} / \mathrm{L}=0$ points $; \leq 1 \mathrm{~g} / \mathrm{L}=1$ point

$\geq 5$ points: compatible with DIC

$<5$ points: non-suggestive for DIC

DIC disseminated intravascular coagulation, ISTH International Society on Thrombosis and Haemostasis

course, preferentially with a DOAC, taking into account possible drug interference.

\section{How to Manage Patients with COVID-19 and Arterial Thrombosis?}

Hospitalizations for ACS have apparently declined during the pandemic [48]. However, it was observed that 20-30\% of patients hospitalized for COVID-19 have a history of cardiovascular disease [49]. As demonstrated in other viral inflammatory syndromes [50], an ACS due to coronary thrombosis can derive from destabilization of pre-existing lesions, as a result of the cytokine storm. Given the paucity of specific data, the pharmacological and interventional management of patients with COVID-19 and ACS should follow the pre-existing guidelines [51], using specific inhospital protocols and appropriate measures for preventing the contagion infecting healthcare workers [52]. As previously indicated, in this setting, antiplatelet strategies play a crucial role. Aspirin can be used without specific additional concerns. In patients with COVID-19 and ACS on lopinavir/ ritonavir or atazanavir treatment, the co-administration of aspirin and prasugrel after PCI should be preferred. If prasugrel is contraindicated, the use of clopidogrel or ticagrelor can be considered, possibly with testing of their antiplatelet efficacy [53]. The metabolism of cangrelor is independent of liver function. No drug interaction is expected between this agent and all drugs used in COVID-19.

Notably, in patients with COVID-19, a high incidence of ACS, with "ST-elevation myocardial infarction (STEMI)like" presentation and without coronary lesions, has been reported [54]. In particular, recent data showed that approximately $40 \%$ of patients with COVID-19 and STEMI did not present culprit lesions at coronary angiography [55]. Possible pathogenetic mechanisms of this finding are acute myocarditis, type 2 myocardial infarction (due to mismatch between oxygen demand and supply), or "Takotsubo-like" myocardiopathy [52]. Thus, in patients with confirmed (or suspected) SARS-CoV-2 infection and "STEMI-like" presentation, fibrinolytic therapy should be considered only in selected cases, when coronary angiography and a possible percutaneous revascularization cannot be performed promptly and safely.

Regarding arterial thrombosis in non-coronary vessels, a recent observational investigation reported a $1.6 \%$ incidence of ischemic stroke among consecutive patients hospitalized for COVID-19 [56]. Moreover, there is evidence that patients with SARS-CoV-2 infection may have a more severe stroke presentation than non-infected patients [57]. Finally, cases of ischemic stroke associated with bilateral acute lower limbs ischemia in the context of antiphospholipid syndrome have been described [58].

\section{How to Manage Patients Hospitalized for COVID-19 Developing Disseminated Intravascular Coagulation?}

Disseminated intravascular coagulation (DIC) represents a possible complication in all patients admitted to the ICU with critical infectious diseases. In patients with COVID-19, a severe clinical course was associated with DIC in $>50 \%$ of cases [45]. Serial evaluation of platelet count, prothrombin time, D-dimer, and fibrinogen plays an important role in diagnosing DIC and monitoring its coagulation abnormalities (Table 4) [59]. The management of these patients includes the identification and treatment of the triggering causes and, in line with international recommendations, the initiation of specific treatments based on platelet transfusion, coagulation factors (fresh concentrated plasma), and fibrinogen (cryo-precipitate) in the presence of bleeding complications or, more rarely, on anticoagulant therapy with heparin, if a thromboembolic pattern is prevalent. In patients with DIC, treatment 
with antiplatelet drugs should be limited to those with recent coronary stent implantation. With regard to patients on dual antiplatelet therapy, such treatment is continued if the platelet count is $\geq 50,000 \times 10^{9} / \mathrm{L}$, whereas if the platelet count is $25,000-50,000 \times 10^{9} / \mathrm{L}$, an antiplatelet agent is stopped, and if the platelet count is $<25,000 \times 10^{9} / \mathrm{L}$, both antiplatelet drugs are withdrawn. However, the management of these patients must be individualized, balancing thrombotic and hemorrhagic risks.

\section{Declarations}

Conflict of interest Giuseppe Patti, Veronica Lio, Ilaria Cavallari, Felice Gragnano, Letizia Riva, Paolo Calabrò, Giuseppe Di Pasquale, Vittorio Pengo, and Andrea Rubboli declare that they have no potential conflicts of interest that might be relevant to the contents of this manuscript.

Funding Open access funding provided by Università degli Studi del Piemonte Orientale Amedeo Avogrado within the CRUI-CARE Agreement. None.

Ethics Approval Not applicable.

Consent to Participate Not applicable.

Consent for Publication Not applicable.

Code Availability Not applicable.

Availability of data and materials Not applicable.

Disclosures GP: Lecture fee/consultant/advisory board for Amgen, Sanofi, Bayer, Boehringer Ingelheim, BMS-Pfizer, Daiichi Sankyo, Astra Zeneca, Sigma-Tau, Malesci, PIAM, and MSD. VL: None. IC: Lecture fee for Boehringer Ingelheim and BMS-Pfizer. FG: None. LR: Lecture fee/consultant/advisory board for Boehringer Ingelheim, Bayer, and Daiichi Sankyo. PC: Lecture fee/consultant/advisory board for Amgen, Sanofi, Bayer, Boehringer Ingelheim, BMS-Pfizer, Daiichi Sankyo, Astra Zeneca, and MSD. GDP: Lecture fee/consultant/advisory board for Boehringer Ingelheim, BMS-Pfizer, Bayer, and Daiichi Sankyo. VP: Lecture fee/consultant/advisory board for Bayer, Daiichi Sankyo, and Werfen Group. AR: Lecture fee/consultant/advisory board for Astra Zeneca, Bayer, Boehringer Ingelheim, Daiichi Sankyo, and Pfizer-BMS.

Open Access This article is licensed under a Creative Commons Attribution-NonCommercial 4.0 International License, which permits any non-commercial use, sharing, adaptation, distribution and reproduction in any medium or format, as long as you give appropriate credit to the original author(s) and the source, provide a link to the Creative Commons licence, and indicate if changes were made. The images or other third party material in this article are included in the article's Creative Commons licence, unless indicated otherwise in a credit line to the material. If material is not included in the article's Creative Commons licence and your intended use is not permitted by statutory regulation or exceeds the permitted use, you will need to obtain permission directly from the copyright holder. To view a copy of this licence, visit http://creativecommons.org/licenses/by-nc/4.0/.

\section{References}

1. Li B, Yang J, Zhao F, et al. Prevalence and impact of cardiovascular metabolic diseases on COVID-19 in China. Clin Res Cardiol. 2020;109(5):531-8.

2. Kirchhof P, Benussi S, Kotecha D, et al. 2016 ESC Guidelines for the management of atrial fibrillation developed in collaboration with EACTS. Eur Heart J. 2016;37:2893-962.

3. Kearon C, Akl EA, Camerota AJ, et al. Antithrombotic therapy for VTE disease: antithrombotic therapy and prevention of thrombosis, 9th ed: American College of Chest Physicians Evidence-Based Clinical Practice Guidelines. Chest. 2012;141(2 Suppl):e419S-494S.

4. Porter AL, Margolis AR, Staresinic CE, et al. Feasibility and safety of a 12 week INR follow-up protocol over 2 years in an anticoagulation clinic: a single-arm prospective cohort study. $\mathrm{J}$ Thromb Thrombolysis. 2019;47:200-8.

5. Plesch W, van den Besselaar AM. Validation of the international normalized ratio (INR) in a new point-of-care system designed for home monitoring of oral anticoagulation therapy. Int J Lab Hematol. 2009;31:20-5.

6. Ruff CT, Giugliano RP, Braunwald E, et al. Comparison of the efficacy and safety of new oral anticoagulants with warfarin in patients with atrial fibrillation: a meta-analysis of randomised trials. Lancet. 2014;383:955-62.

7. Meyer S, Frei CR, Kelly RD. Impact of a new method of warfarin management on patient satisfaction, time and cost. Pharmacotherapy. 2013;33:1147-55.

8. Mahé I, Bertrand N, Drouet L, et al. Interaction between paracetamol and warfarin in patients: a double-blind, placebocontrolled, randomized study. Haematologica. 2006;91:1621-7.

9. European Society of Cardiology. ESC guidance for the diagnosis and management of CV disease during the COVID-19 pandemic. Bruxelles: European Society of Cardiology; 2020.

10. Misra S, Nath M, Hadda V, Vibha D. Efficacy of various treatment modalities for nCOV-2019: a systematic review and metaanalysis. Eur J Clin Invest. 2020;e13383.

11. Sattui SE, Liew JW, Graef ER, et al. Swinging the pendulum: lessons learned from public discourse concerning hydroxychloroquine and COVID-19. Expert Rev Clin Immunol. 2020;16:659-66.

12. de Wilde AH, Jochmans D, Posthuma CC, et al. Screening of an FDA-approved compound library identifies four small-molecule inhibitors of Middle East respiratory syndrome coronavirus replication in cell culture. Antimicrob Agents Chemother. 2014;58:4875-84.

13. Chan JF, Yao Y, Yeung ML, et al. Treatment with lopinavir/ ritonavir or interferon- $\beta 1 \mathrm{~b}$ improves outcome of MERSCoV infection in a nonhuman primate model of common marmoset. J Infect Dis. 2015;212:1904-13.

14. Arabi YM, Alothman A, Balkhy HH, et al. Treatment of Middle East respiratory syndrome with a combination of lopinavirritonavir and interferon- $\beta 1 \mathrm{~b}$ (MIRACLE trial): study protocol for a randomized controlled trial. Trials. 2018;19:81.

15. Park SY, Lee JS, Son JS, et al. Post-exposure prophylaxis for Middle East respiratory syndrome in healthcare workers. J Hosp Infec. 2019;101:42-6.

16. Cao B, Wang Y, Wen D, et al. A trial of lopinavir-ritonavir in adults hospitalized with severe Covid-19. N Engl J Med. 2020;382(19):1787-99.

17. Sheahan TP, Sims AC, Graham RL, et al. Broad-spectrum antiviral GS-5734 inhibits both epidemic and zoonotic coronaviruses. Sci Transl Med. 2017;9:eaa13653. 
18. de Wit E, Feldmann F, Cronin J, et al. Prophylactic and therapeutic remdesivir (GS-5734) treatment in the rhesus macaque model of MERS-CoV infection. Proc Natl Acad Sci USA. 2020;117:6771-6.

19. Sheahan TP, Sims AC, Leist SR, et al. Comparative therapeutic efficacy of remdesivir and combination lopinavir, ritonavir, and interferon beta against MERS-CoV. Nat Commun. 2020;11:222.

20. 20Jiang Y, Chen D, Cai D, Yi Y, Jiang S. Effectiveness of remdesivir for the treatment of hospitalized Covid-19 persons: a network meta-analysis. J Med Virol. 2020.

21. Khalili JS, Zhu H, Mak NSA, Yan Y, Zhu Y. Novel coronavirus treatment with ribavirin: groundwork for evaluation concerning COVID-19. J Med Virol. 2020;92:740-6.

22. Arshad S, Kilgore P, Chaudhry ZS, et al. Treatment with hydroxychloroquine, azithromycin, and combination in patients hospitalized with COVID-19. Int J Infect Dis. 2020;S1201-9712(20):30534-8.

23. The RECOVERY Collaborative Group. Dexamethasone in hospitalized patients with Covid-19-preliminary report. N Engl J Med. 2020. https://doi.org/10.1056/NEJMoa2021436.

24. Luo P, Liu Y, Qiu L, Liu X, Liu D, Li J. Tocilizumab treatment in COVID-19: a single center experience. J Med Virol. 2020;92(7):814-8.

25. Patti G, Lio V, Cavallari I, et al. Antithrombotic treatments in patients with SARS-CoV-2 infection: from current evidence to reasonable recommendations-a position paper from the Italian Working Group on Atherosclerosis, Thrombosis and Vascular Biology. G Ital Cardiol (Rome). 2020;21(7):489-501.

26. Raskob GE, van Es N, Verhamme P, et al. Edoxaban for the treatment of cancer-associated venous thromboembolism. N Engl J Med. 2018;378:615-24.

27. Testa S, Prandoni P, Paoletti O, et al. Direct oral anticoagulant plasma levels' striking increase in severe COVID-19 respiratory syndrome patients treated with antiviral agents: the Cremona experience. J Thromb Haemost. 2020;18(6):1320-3.

28. Mahamid M, Mader R, Safadi R. Hepatotoxicity of tocilizumab and anakinra in rheumatoid arthritis: management decisions. Clin Pharmacol. 2011;3:39-433.

29. Russell B, Moss C, Rigg A, Van Hemelrijck M. COVID-19 and treatment with NSAIDs and corticosteroids: should we be limiting their use in the clinical setting? Ecancermedicalscience. 2020;14:1023.

30. McCullough PA, Kelly RJ, Ruocco G, et al. Pathophysiological basis and rationale for early outpatient treatment of SARS-CoV-2 (COVID-19) Infection. Am J Med. 2020;S0002-9343(20):30673-82.

31. Ford N, Vitoria M, Rangaraj A, Norris SL, Calmy A, Doherty M. Systematic review of the efficacy and safety of antiretroviral drugs against SARS, MERS or COVID-19: initial assessment. J Int AIDS Soc. 2020;23(4):e25489.

32. Singhania N, Bansal S, Nimmatoori DP, et al. Current overview on hypercoagulability in COVID-19. Am J Cardiovasc Drugs. 2020;1-11.

33. Bikdeli B, Madhavan MV, Jimenez D, et al. COVID-19 and thrombotic or thromboembolic disease: implications for prevention, antithrombotic therapy, and follow-up. J Am Coll Cardiol. 2020;75(23):2950-73.

34. Zhai Z. Prevention and treatment of venous thromboembolism associated with coronavirus disease 2019 infection: a consensus statement before guidelines. Thromb Haemost. 2020;120(6):937-48.

35. Barbar S, Noventa F, Rossetto V, et al. A risk assessment model for the identification of hospitalized medical patients at risk for venous thromboembolism: the Padua Prediction Score. J Thromb Haemost. 2010;8:2450-7.
36. Rosenberg D, Eichorn A, Alarcon M, McCullagh L, McGinn T, Spyropoulos AC. External validation of the risk assessment model of the International Medical Prevention Registry on Venous Thromboembolism (IMPROVE) for medical patients in a tertiary health system. J Am Heart Assoc. 2014;3:e001152.

37. World Health Organization. Clinical management of severe acute respiratory infection when novel coronavirus $(2019 \mathrm{nCoV})$ infection is suspected. Interim guidance. 2020. https://www.who.int/ publications-detail/clinical-management-of-severe-acute-respi ratory-infection-when-novel-coronavirus-(ncov)-infection-issuspected. Accessed 31 July 2020.

38. Tang N, Bai H, Chen X, Gong J, Li D, Sun Z. Anticoagulant treatment is associated with decreased mortality in severe coronavirus disease 2019 patients with coagulopathy. J Thromb Haemost. 2020;18(5):1094-9.

39. Paranjpe I, Fuster V, Lala A, Russak A, Glicksberg BS, Levin MA, Charney AW, Narula J, Fayad ZA, Bagiella E, Zhao S, Nadkarni GN. Association of treatment dose anticoagulation with inhospital survival among hospitalized patients with COVID-19. J Am Coll Cardiol. 2020;76:122-4.

40. Zhou F, Yu T, Du R, et al. Clinical course and risk factors for mortality of adult inpatients with COVID-19 in Wuhan, China: a retrospective cohort study. Lancet. 2020;395:1054-62.

41. Konstantinides SV, Meyer G, Becattini C, et al. 2019 ESC Guidelines for the diagnosis and management of acute pulmonary embolism developed in collaboration with the European Respiratory Society (ERS). Eur Heart J. 2020;41:543-603.

42. Rotzinger DC, Beigelman-Aubry C, von Garnier C, Qanadli SD. Pulmonary embolism in patients with COVID-19: time to change the paradigm of computed tomography. Thromb Res. 2020;190:58-9.

43. Lippi G, Plebani M. Laboratory abnormalities in patients with COVID-2019 infection. Clin Chem Lab Med. 2020;58:1131-4.

44. Stefanini GG, Chiarito M, Ferrante G, et al. Early detection of elevated cardiac biomarkers to optimise risk stratification in patients with COVID-19. Heart. 2020;106:1512-8.

45. Tang N, Li D, Wang X, Sun Z. Abnormal coagulation parameters are associated with poor prognosis in patients with novel coronavirus pneumonia. J Thromb Haemost. 2020;18:844-7.

46. Clerkin KJ, Fried JA, Raikhelkar J, et al. COVID-19 and cardiovascular disease. Circulation. 2020;141:1648-55.

47. Onder G, Rezza G, Brusaferro S. Case-fatality rate and characteristics of patients dying in relation to COVID-19 in Italy. JAMA. 2020;323:1775-6.

48. De Filippo O, D’Ascenzo F, Angelini F, et al. Reduced rate of hospital admissions for ACS during Covid-19 outbreak in Northern Italy. N Engl J Med. 2020;383:88-9.

49. Kang Y, Chen T, Mui D. Cardiovascular manifestations and treatment considerations in covid-19. Heart. 2020;106:1132-41.

50. Kwong JC, Schwartz KL, Campitelli MA, et al. Acute myocardial infarction after laboratory-confirmed influenza infection. $\mathrm{N}$ Engl J Med. 2018;378:2540-1.

51. Neumann FJ, Sousa-Uva M, Ahlsson A, et al. 2018 ESC/ EACTS Guidelines on myocardial revascularization. Eur Heart J. 2019;40:87-165.

52. Scotto di Uccio F, Valente S, Colivicchi F, et al. Position paper ANMCO: Organizzazione della Rete per il trattamento dei pazienti con sindrome coronarica acuta durante emergenza pandemica COVID-19. G Ital Cardiol. 2020;21:332-5.

53. Driggin E, Madhavan MV, Bikdeli B, et al. Cardiovascular considerations for patients, health care workers, and health systems during the coronavirus disease 2019 (COVID-19) pandemic. J Am Coll Cardiol. 2020;75:2352-71.

54. Shi S, Qin M, Shen B, et al. Association of cardiac injury with mortality in hospitalized patients with COVID-19 in Wuhan. China. JAMA Cardiol. 2020;5:802-10. 
55. Stefanini GG, Montorfano M, Trabattoni D, et al. ST-elevation myocardial infarction in patients with COVID-19: Clinical and angiographic outcomes. Circulation. 2020;141:2113-6.

56. Bilaloglu S, Aphinyanaphongs $\mathrm{Y}$, Jones $\mathrm{S}$, et al. Thrombosis in Hospitalized Patients With COVID-19 in a New York City Health System. JAMA. 2020;324:799-801.

57. Escalard S, Chalumeau V, Escalard C, et al. Early brain imaging shows increased severity of acute ischemic strokes with large vessel occlusion in COVID-19 Patients. Stroke. 2020. https://doi. org/10.1161/STROKEAHA.120.031011.

58. Zhang Y, Xiao M, Zhang S, et al. Coagulopathy and antiphospholipid antibodies in patients with Covid-19. N Engl J Med. 2020;382:e38.

59. Levi M, Toh CH, Thachil J, Watson HG. Guidelines for the diagnosis and management of disseminated intravascular coagulation. Br J Haematol. 2009;145:24-33. 